\title{
The Happy Survivor? Effects of Differential Mortality on Life Satisfaction in Older Age
}

\author{
Suzanne C. Segerstrom, \\ Department of Psychology, University of Kentucky \\ Hannah L. Combs, \\ Department of Psychology, University of Kentucky \\ Ashley Winning, \\ Department of Social and Behavioral Sciences, Harvard School of Public Health, Harvard \\ University \\ Julia K. Boehm, and \\ Department of Psychology, Chapman University

\section{Laura D. Kubzansky} \\ Department of Social and Behavioral Sciences, Harvard T.H. Chan School of Public Health, \\ Harvard University.
}

\section{Abstract}

Older adults report higher psychological well-being than younger adults. Those highest in wellbeing also have the lowest risk of mortality. If those with lower well-being die earlier, it could affect the appearance of developmental change in well-being. In adults aged 50 and older $(\mathrm{N}=4,458)$, we estimated effects of differential mortality on life satisfaction by imputing life satisfaction, adjusting for attrition due to death, or estimating life satisfaction using patternmixture modeling. There was an increase in life satisfaction with age; however, differential mortality affected the elevation of the curve. Observed life satisfaction, particularly above age 70 , is affected by differential mortality.

\section{Keywords}

life satisfaction; well-being; mortality; aging

Older adults, when compared with younger adults, report more positive affect and less negative affect, report more motivation to maintain positive affect, and have positive memory and attention biases (e.g., Brassen, Gamer, \& Buchel, 2011; Isaacowitz, Toner, Goren, \& Wilson, 2008; Murphy \& Isaacowitz, 2008; Riedeger, Schmiedek, Wagner, \& Linderberger, 2009). These phenomena are consistent with socioemotional selectivity theory (Carstensen, Isaacowitz, \& Charles, 1999), which proposes that when remaining time in life

Correspondence concerning this article should be addressed to Suzanne C. Segerstrom, Department of Psychology, University of Kentucky, 125 Kastle Hall, Lexington, KY, 40506-0044. segerstrom@uky.edu. 
is perceived as limited, as in older age, motivation and goals shift toward maintaining positive affect. Longitudinal studies support increases in psychological well-being into older age that are qualified by a "terminal decline" in which well-being begins to decrease in the months to years prior to death (Carstensen et al., 2011; Charles, Reynolds, \& Gatz, 2001; Gerstorf, Ram, Estabrook, et al., 2008; Gerstorf, Ram, Röcke et al., 2008; Gerstorf, et al., 2010; Holahan, Holahan, Velasquez, \& North, 2008; Mroczek \& Spiro, 2005; Yang, 2007).

Longitudinal studies also reveal another phenomenon: those highest in well-being also have the lowest subsequent risk of mortality (Boehm et al., 2015; Carstensen et al., 2011; Chida $\&$ Steptoe, 2008). If older adults with lower well-being die earlier than those with higher well-being, differential mortality should affect how age differences in well-being and associated cognitive and social processes are interpreted. For example, in the presence of differential mortality, one might attribute lower well-being in younger adults compared with older adults to the prior death of individuals with low well-being among the older adults rather than to developmental change. In longitudinal studies, there are established methods for estimating and accounting for differential mortality. However, in more common crosssectional studies, the effect of differential mortality would not be obvious, and it is this effect that we wish to explore.

Of note, it is important to recognize that we are not necessarily arguing that mortality causes a change in life satisfaction among surviving individuals; instead, it may appear that change occurs because a different group of individuals remain available for assessment (i.e., those with higher levels of well-being) once decedents are no longer available. To illustrate how differential mortality might affect the distribution of well-being in the surviving population and the degree to which it does so, it is useful to consider two factors: first, the strength of the relationship between well-being and mortality risk, and second, the mortality rate. First, the stronger the relationship between well-being and mortality risk, the greater the difference there will be between decedents and survivors. For example, if there is a strong relationship between well-being and mortality risk, the people who die will have markedly lower wellbeing than the people who survive. If there is a weak relationship, the people who die will have subtly lower well-being than the people who survive. The ultimate effect of this relationship is then determined by the mortality rate. If few people in the population die, that is, the mortality rate is low, then there will be little impact on the average well-being of the surviving population. As an extreme example, if 1 person out of 10,000 dies, even if that person has extremely low well-being, the average well-being of the survivors will not be affected very much. Therefore, the degree to which differential mortality among older adults affects observed well-being will be population-dependent: When relationships are stronger and mortality is higher, effects of differential mortality will be greater; when relationships are weaker and mortality is lower, effects of differential mortality will be lesser.

The present study investigated the effect of differential mortality on one aspect of positive well-being, life satisfaction, in older adulthood. Life satisfaction is a cognitive-judgmental measure of well-being that reflects satisfaction with one's present life compared with one's own standards; as such, it is "a hallmark of the subjective well-being area" (Diener et al., 1985, p. 71). We sought to address two questions in the study sample: First, what would life satisfaction look like across older adulthood if not for differential mortality? That is, what 
would hypothetical life satisfaction be if everyone in the sample survived, not just those with higher life satisfaction? Second, at what age does observed life satisfaction in older adults depart from hypothetical life satisfaction if everyone in the sample survived? That is, at what age might observed life satisfaction be expected to be affected by differential mortality?

To answer these questions, we employed a subsample from the Household, Income, and Labour Dynamics in Australia (HILDA) Survey, which assessed a national probability sample of Australian households. Recall that the degree to which differential mortality among older adults affects observed well-being will depend on the strength of the relationship and the mortality rate. In the present sample, we previously found a relationship between life satisfaction and mortality risk: Each standard deviation higher life satisfaction was associated with an $18 \%$ decrease in mortality risk (hazard ratio $=0.82$ ) after adjusting for age. The relationship was partially accounted for by health conditions and behaviors (Boehm et al., 2015; see Table 1). The magnitude and direction of this hazard estimate was consistent with meta-analytic findings for positive well-being and mortality risk in healthy samples (meta-analytic hazard ratio $=.82$; Chida \& Steptoe, 2008), suggesting that this HILDA subsample was representative in terms of this contributor to differential mortality. The overall mortality over the 9-year study period was $12 \%$.

We used three strategies to evaluate how differential mortality might influence observed levels of life satisfaction in older adulthood. First, we imputed life satisfaction for individuals who died over the 9-year follow-up. Second, we applied an algorithm that adjusts for differential mortality. Third, we estimated life satisfaction over time from a pattern-mixture model. Exploratory analyses investigated whether differential mortality differed between men and women, who differ in both mortality rates and levels of life satisfaction.

\section{Method}

\section{Participants and procedure}

Data were from the Household, Income, and Labour Dynamics in Australia (HILDA) Survey, which assessed a national probability sample of Australian households. HILDA initially included 19,914 individuals who were followed for up to 9 years with annual assessments. The subsample for analysis was that reported in Boehm et al. (2015), with its known relationship between life satisfaction and mortality risk. This sample excluded individuals less than 50 years of age $(\mathrm{N}=14,789)$ because $91 \%$ of mortality in HILDA occurred at age 50 or older, and in the absence of mortality, there is no differential mortality. The sample also excluded individuals with only one assessment of life satisfaction because they could not contribute to change estimates $(\mathrm{N}=667)$; these individuals were more likely to be male, less educated, and physically inactive than the analytic sample (Boehm et al., 2015). The analytic subsample $(\mathrm{N}=4,458)$ was $53 \%$ female and had an average age at study inception of 63 years $(S D=10$, minimum $=50$, maximum $=92)$. HILDA participants were interviewed either face-to-face or by telephone (when face-to-face interviews could not be conducted) annually between 2001 and 2010, with each participant interviewed up to nine times. All participants provided informed consent. 
At each interview wave, HILDA participants were asked, "All things considered, how satisfied are you with your life?" with possible responses ranging from $0=$ "totally dissatisfied" to $10=$ "totally satisfied"; $89 \%$ of responses were between 7 and 10 . This single-item measure provides an adequately reliable measure of life satisfaction (in the full HILDA sample, "true" or reliable variance ranged from 63\% to 73\%; Lucas \& Donnellan, 2012). Mortality and date of death were confirmed by interviews with surviving members of the household or other contacts.

\section{Statistical analyses}

We used three methods to compare observed life satisfaction across late middle and older age with hypothetical life satisfaction after accounting for differential mortality. First, we used modified multiple imputation techniques (taking the first step in multiple imputation procedure to develop stable imputes or substitutes for the missing values) to estimate what life satisfaction might have been for individuals who were missing data due to death. Second, we estimated the effect of differential mortality using an adjustment described by Salthouse (2010). Third, we used pattern-mixture modeling to estimate life satisfaction considering survival or death (Hedeker \& Gibbons, 1997). We therefore illustrate three trajectories of life satisfaction: an "observed" trajectory in which only intermittently missing data (e.g., missing at Wave 4, but not Wave 3 or Wave 5) were imputed, an "imputed" trajectory in which life satisfaction was also imputed after death, an "adjusted" trajectory that accounted for the effect of attrition due to mortality on life satisfaction at each wave, and an "estimated" trajectory in which life satisfaction was estimated after death from the parameters of a pattern-mixture model.

Analyses accounting for clustering by household (reported in Boehm et al., 2015) did not yield any differences in the relationship between life satisfaction and mortality compared with analyses not doing so. Therefore, differential mortality would also not be affected by household clusters, and this level of analysis was not considered further in the present study.

Describing the trajectory of life satisfaction-A statistical description of the observed life satisfaction trajectory over time was generated using multi-level modeling (SAS PROC MIXED; Singer, 1998), with individuals at Level 2 and observations at Level 1. Age was centered around 50 years for the growth curve model. Gamma weights (analogous to unstandardized beta weights) are reported with their empirical standard errors.

Imputing missing data—Life satisfaction was missing intermittently (e.g., missing at Wave 6, but not Wave 5 or Wave 7; $2.3 \%$ of data), from dropout (e.g., missing from Wave 6 through the end of the study, but not due to death between Wave 6 and Wave 7; 6.7\% of data), and from death (e.g., missing from Wave 6 through the end of the study due to death between Wave 6 and Wave 7; 6.0\% of data). We imputed missing data using multiple imputation in IBM SPSS Version 22.0. SPSS utilizes a Markov chain Monte Carlo (MCMC) algorithm known as fully conditional specification (FCS), or chained equations imputation. This approach imputes incomplete variables one at a time through linear regression, using the non-missing variable from one step as a predictor in all subsequent steps. All missing data were imputed in the "wide" data set (i.e., each participant had one record with multiple 
observations of life satisfaction) on the basis of decedent status (decedents, $\mathrm{N}=546$; survivors, $N=3,912$ ) and available life satisfaction scores. To reduce the potential effect of terminal decline on the projection of hypothetical future life satisfaction, the last available assessment of life satisfaction was not used for imputation for decedents. For the "imputed" dataset, we took the average of 5 imputations to replace missing life satisfaction data. When a minority of the data are missing, estimates based on 5 imputations are very close to those with 100 or more imputations (Graham, Olchowski, \& Gilreath, 2007; Schafer, 1999).

Adjusting for differential mortality-Salthouse (2010) accounted for effects of attrition by calculating the attrition adjustment term $A$ : the mean score of the total sample at one assessment subtracted from the mean of the longitudinal sample (those who continued to the next assessment). This value represents the selectivity of the continuing sample relative to the total sample at each subsequent assessment. Beginning at age 51, $A$ values associated with each year of age were added to the observed life satisfaction mean at each subsequent year of age (e.g., from age 57 to 58,58 to 59, etc.) in order to obtain an adjusted estimate of life satisfaction at each year of age. We calculated $A$ separately for dropout and death. Attrition due to dropout was associated with minimal differences: The results of a Tukey HSD test indicated that across all ages, people who dropped out did not have reliably different life satisfaction scores than those who remained in the study $(M D=-0.165, S E=$. $090, p=.166)$. In contrast, death was associated with more substantial differences $(M D=$ $-0.518, S E=.090, p<.001)$. Therefore, for the "adjusted" life satisfaction trajectory presented below, the adjustment is primarily driven by effects of attrition due to death.

Pattern mixture modeling-We fitted a pattern mixture model in which linear and quadratic age were moderated by dummy variables indicating dropout due to death at each wave at which death occurred. As in the imputation, we did not use the last available assessment of life satisfaction among decedents to reduce the potential effects of terminal decline on the estimates. Addition of the pattern-mixture parameters significantly improved the model $\left(\chi^{2}(24)=45.0, p=.0058\right)$. The model fixed parameters were then used to estimate life satisfaction where missing due to death. For example, for a person dying after Wave 4, life satisfaction from Waves 4 to 9 was estimated by the following parameters: intercept, linear age (i.e., the age the person would have been at each wave from 4 to 9), quadratic age, the effect associated with the pattern (death after Wave 4), and the interactions between that pattern, age, and quadratic age.

\section{Results}

\section{Descriptive analysis}

Using the data unadjusted for mortality (i.e., imputing only intermittently missing data), an unconditional means model with no predictors (Singer \& Willett, 2003) estimated the sample life satisfaction mean across all waves (i.e., the intercept) at 8.18 (SE = 0.02), illustrating the generally high life satisfaction in this sample. The intraclass correlation was . 52 , indicating that $52 \%$ of the variability in life satisfaction was due to individual differences, with $48 \%$ of the variability due to differences within people across waves. An unconditional growth model added age and quadratic age as predictors, allowing the effect 
of age to vary across people (i.e., a random effect of age). The intercept (at age 50) in this model was 7.88 ( $\mathrm{SE}=0.05)$. There was a significant increase in life satisfaction with each additional year of age $(\gamma=0.043, \mathrm{SE}=0.005, p<.0001$; slope variance $=0.0026, \mathrm{SE}=$ $0.00027)$ that was moderated by a significant quadratic effect describing a downturn in life satisfaction at older ages $(\gamma=-0.0011, \mathrm{SE}=0.00013, p<.0001)$.

\section{Effects of mortality on mean life satisfaction}

Figure 1 shows mean life satisfaction at each year of age from 55 to 90 , where $96 \%$ of the deaths occurred and where effects of differential mortality were present and life satisfaction means had small standard errors. (There were no deaths affecting estimates before age 55 and therefore no differential mortality, and small sample sizes above age 90, yielding larger standard errors.) Because we intend to illustrate the potential effect of differential mortality in cross-sectional designs (or longitudinal designs in which differential mortality is not modeled), we present the observed mean life satisfaction in each year, which is comparable to a cross-sectional result. The solid line in Figure 1 with standard errors shows the observed data that included imputations only for intermittently missing life satisfaction. The longdash line in Figure 1 shows mean life satisfaction in each year after imputing life satisfaction when it was missing due to death. The short-dash line in Figure 1 shows mean life satisfaction in each year after adjustment for differential mortality. The broken long-dash line shows mean life satisfaction in each year after estimating life satisfaction from the pattern-mixture model when it was missing due to death. These methods agree well with each other in terms of the effect of differential mortality. Estimates began to fall outside the standard error of the observed values among people 60 years old or older and became consistently lower than observed mean levels among people in their 70s. Means and standard errors for all ages and methods are found in the Supplemental Online Material.

Figure 2 shows the differences between observed mean life satisfaction and imputed and adjusted life satisfaction levels expressed as Cohen's $d$ (the difference between the observed mean and the imputed, adjusted, and estimated means, all in observed SD units so they are expressed on the same scale). In general, the effect sizes increased with increasing age (and increasing number of deaths), but were negligible ( $d \mathrm{~s}<.05$ in the decade of the $50 \mathrm{~s}$ ) to small ( $d$ s up to .10 in the decades of the 70 s and 80 s).

\section{Gender differences}

Between ages 55 and 90 (the range illustrated in Figures 1 and 2), there were somewhat more deaths among men $(\mathrm{N}=302 ; 14 \%)$ than women $(\mathrm{N}=221 ; 9 \%)$. The degree of differential mortality was similar across genders: There was no systematic relationship between gender and magnitude of differential mortality across ages and methods of calculation (see Supplemental Online Material). However, the correlation between differential mortality (the difference between the observed mean and average hypothetical mean) and number of deaths at each age was higher among men $(r=.37)$ than among women $(r=.22)$. The larger correlation among men suggests that differential mortality could be driven more strongly by mortality rate among men and more strongly by the strength of the association between life satisfaction and mortality risk among women. 


\section{Discussion}

Higher levels of positive affect and positive dispositions such as life satisfaction have been linked to lower mortality risk (Chida \& Steptoe, 2008). Observed levels of life satisfaction and other measures of well-being increase with age until shortly prior to death. However, observed levels are also affected by the phenomenon in which, with increasing age, the population of older adults changes due to differential mortality: Those who have survived are those with higher levels of well-being. This raises the question of whether life satisfaction and well-being increase with age as a developmental process, the apparent increase is an artifact of a healthy survivor effect, or some combination thereof. The present investigation was meant to explore this issue and to evaluate what life satisfaction might look like in older age if differential mortality did not occur.

Regardless of the data used (observed, imputed for those who died, adjusted for differential mortality, estimated for those who died), there was a modest increase in life satisfaction with age that was increasingly attenuated with older age (see Figure 1). This trajectory replicates the findings of prior longitudinal studies of affect and life satisfaction (Carstensen et al., 2011; Charles et al., 2001; Holahan et al., 2008; Mroczek \& Spiro, 2005) and further indicates that apparent increases in life satisfaction and, possibly, other positive psychological characteristics in older age are not due solely to differential mortality. However, it is worth noting that differential mortality did affect the elevation of the curve. We projected what life satisfaction might have been if those who died had continued to contribute life satisfaction ratings by imputing their life satisfaction ratings, adjusting life satisfaction means for differential mortality, or estimating life satisfaction from a patternmixture model. The findings suggested that among older adults in their 70s and 80s, observed increases in life satisfaction may have been due in part to differential mortality, consistent with the link between low life satisfaction and increased mortality risk in this sample (Table 1; Boehm et al., 2015). Because individuals who died tended to have lower life satisfaction, their absence from the observed sample created a degree of elevation in life satisfaction that was not due to developmental change. This elevation was on the order of 0.1 scale units. Although this difference is small in the context of the total range of the life satisfaction scale (0-10), it is more meaningful in the context of the range of the scale that most participants employed (7-10), and particularly in the context of the change in life satisfaction across older adulthood (approximately 0.5 scale points; note that this change was similar to that in, e.g., negative affect; Charles et al., 2001). Therefore, although small in an absolute sense, the effect of differential mortality may be meaningful in the context of interpreting differences in life satisfaction involving older adults.

A clear limitation of this investigation is that it is impossible to know with certainty what a person's life satisfaction would have been had he or she not died; we can only surmise. We recognize that projection of life satisfaction into a hypothetical future is somewhat arbitrary. For example, one possibility is that approaching death suppressed life satisfaction in the deceased group (even after excluding their last measure of life satisfaction; Gerstorf, Ram, Estabrook, et al., 2008; Gerstorf, Ram, Röcke et al., 2008; Gerstorf, et al., 2010). This cannot be the only explanation, however, because even the first assessment of life satisfaction (typically measured more than 3 years before death) predicted lower mortality 
risk in this sample, with little effect on the hazard ratio (Boehm et al., 2015). A degree of confidence is also gained by good agreement between the different methods of estimating the effect of differential mortality. Another limitation is that findings may not generalize beyond this specific sample to other samples that differ in culture, physical health, or other important attributes. The present sample was in generally good health (e.g., half of the sample engaged in regular physical activity, a proportion higher than in other older adult samples; Boehm et al., 2015; Hallal et al., 2012). Therefore, mortality rates in this sample were lower than one might expect in less healthy samples. Note that higher mortality rates would result in larger effects of differential mortality, keeping the relationship between life satisfaction and mortality risk constant.

In summary, we replicated the general shape of the trajectory of life satisfaction in older age previously reported, but we caution against making inferences about the effect of aging on well-being in the very old without taking into account differential mortality; apparent associations between aging and enhanced well-being may be partly a function of happy survivor effects.

\section{Supplementary Material}

Refer to Web version on PubMed Central for supplementary material.

\section{Acknowledgments}

Support was provided by the National Institute of Aging (K02-033629) and the Robert Wood Johnson Foundation ("Exploring the Concept of Positive Health," to the Positive Psychology Center of the University of Pennsylvania, Martin Seligman, project director). The Household, Income and Labour Dynamics in Australia Survey was initiated and is funded by the Australian Government Department of Families, Housing, Community Services and Indigenous Affairs (FaHCSIA) and is managed by the Melbourne Institute of Applied Economic and Social Research (Melbourne Institute). The findings and views reported in this paper are those of the authors and should not be attributed to either FaHCSIA or the Melbourne Institute. The authors thank Brent Roberts for contributions to the conception of this project and his helpful comments on this paper.

\section{References}

Boehm JK, Winning A, Segerstrom SC, Kubzansky LD. Variability modifies life satisfaction's association with mortality risk in older adults. Psychological Science. 2015; 26:1063-1070. [PubMed: 26048888]

Brassen S, Gamer M, Buchel C. Anterior cingulate activity is related to a positivity bias and emotional stability in successful aging. Biological Psychiatry. 2011; 70:131-137. [PubMed: 21183158]

Carstensen LL, Isaacowitz DM, Charles ST. Taking time seriously: A theory of socioemotional selectivity. American Psychologist. 1999; 54:165-181. [PubMed: 10199217]

Carstensen LL, Turan B, Scheibe S, Ram N, Ersner-Hershfield H, Samanez-Larkin GR, Nesselroade JR. Emotional experience improves with age: evidence based on over 10 years of experience sampling. Psychology and Aging. 2011; 26:21-33. [PubMed: 20973600]

Charles ST, Reynolds CA, Gatz M. Age-related differences and change in positive and negative affect over 23 years. Journal of Personality and Social Psychology. 2001; 80:136-151. [PubMed: 11195886]

Chida Y, Steptoe A. Positive psychological well-being and mortality: a quantitative review of prospective observational studies. Psychosomatic Medicine. 2008; 70:741-756. [PubMed: 18725425]

Diener ED, Emmons RA, Larsen RJ, Griffin S. The Satisfaction with Life Scale. Journal of Personality Assessment. 1985; 49:71-75. [PubMed: 16367493] 
Gerstorf D, Ram N, Estabrook R, Schupp J, Wagner GG, Lindenberger U. Life satisfaction shows terminal decline in old age: longitudinal evidence from the German Socio-Economic Panel Study (SOEP). Developmental Psychology. 2008; 44:1148-1159. [PubMed: 18605841]

Gerstorf D, Ram N, Mayraz G, Hidajat M, Lindenberger U, Wagner GG, Schupp J. Late-life decline in well-being across adulthood in Germany, the United Kingdom, and the United States: Something is seriously wrong at the end of life. Psychology and Aging. 2010; 25:477-485. [PubMed: 20545432]

Gerstorf D, Ram N, Röcke C, Lindenberger U, Smith J. Decline in life satisfaction in old age: longitudinal evidence for links to distance-to-death. Psychology and Aging. 2008; 23:154-168. [PubMed: 18361663]

Graham JW, Olchowski AE, Gilreath TD. How many imputations are really needed? Some practical clarifications of multiple imputation theory. Prevention Science. 2007; 8:206-213. [PubMed: 17549635]

Hallal PC, Andersen LB, Bull FC, Guthold R, Haskell W, Ekelund U. Global physical activity levels: surveillance progress, pitfalls, and prospects. The Lancet. 2012; 380(9838):247-257.

Hedeker D, Gibbons RD. Application of random-effects pattern-mixture models for missing data in longitudinal studies. Psychological Methods. 1997; 2:64-78.

Holahan CK, Holahan CJ, Velasquez KE, North RJ. Longitudinal change in happiness during aging: The predictive role of positive expectancies. The International Journal of Aging and Human Development. 2008; 66:229-241. [PubMed: 18459603]

Isaacowitz DM, Toner K, Goren D, Wilson HR. Looking while unhappy: Mood-congruent gaze in young adults, positive gaze in older adults. Psychological Science. 2008; 19:848-853. [PubMed: 18947348]

Lucas RE, Donnellan MB. Estimating the reliability of single-item life satisfaction measures: Results from four national panel studies. Social Indicators Research. 2012; 105:323-331. [PubMed: 23087538]

Mroczek DK, Spiro A. Change in life satisfaction during adulthood: findings from the Veterans Affairs Normative Aging Study. Journal of Personality and Social Psychology. 2005; 88:189-202. [PubMed: 15631584]

Murphy NA, Isaacowitz DM. Preferences for emotional information in older and younger adults: A met-analysis of memory and attention tasks. Psychology and Aging. 2008; 23:263-286. [PubMed: 18573002]

Riediger M, Schmiedek F, Wagner GG, Linderberger U. Seeking pleasure and seeking pain: Differences in prohedonic and contra-hedonic motivation from adolescence to old age. Psychological Science. 2009; 20:1529-1535. [PubMed: 19891749]

Salthouse TA. Influence of age on practice effects in longitudinal neurocognitive change. Neuropsychology. 2010; 24:563-572. [PubMed: 20804244]

Schafer JL. Multiple imputation: A primer. Statistical Methods in Medical Research. 1999; 8:3-15. [PubMed: 10347857]

Singer JD. Using SAS PROC MIXED to fit multilevel models, hierarchical models, and individual growth models. Journal of Educational and Behavioral Statistics. 1998; 23:323-355.

Singer, JD.; Willett, JB. Applied longitudinal data analysis: Modeling change and event occurrence. Oxford University Press; New York: 2003.

Yang Y. Is old age depressing? Growth trajectories and cohort variations in late-life depression. Journal of Health and Social Behavior. 2007; 48:16-32. [PubMed: 17476921] 


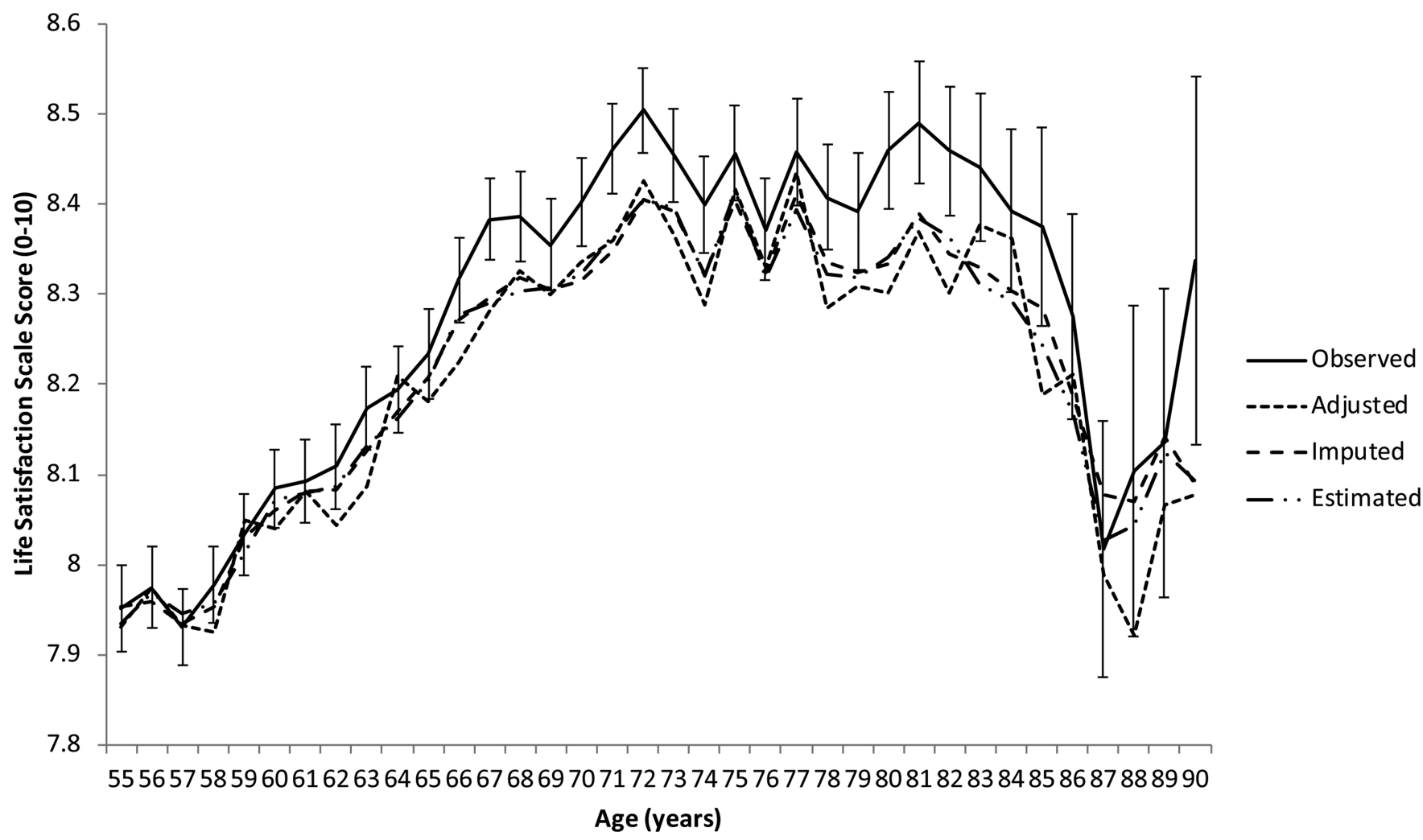

Figure 1.

Observed mean life satisfaction at each year of age (with standard error) and predicted life satisfaction when differential mortality was accounted for by imputation, estimation, or adjustment. 


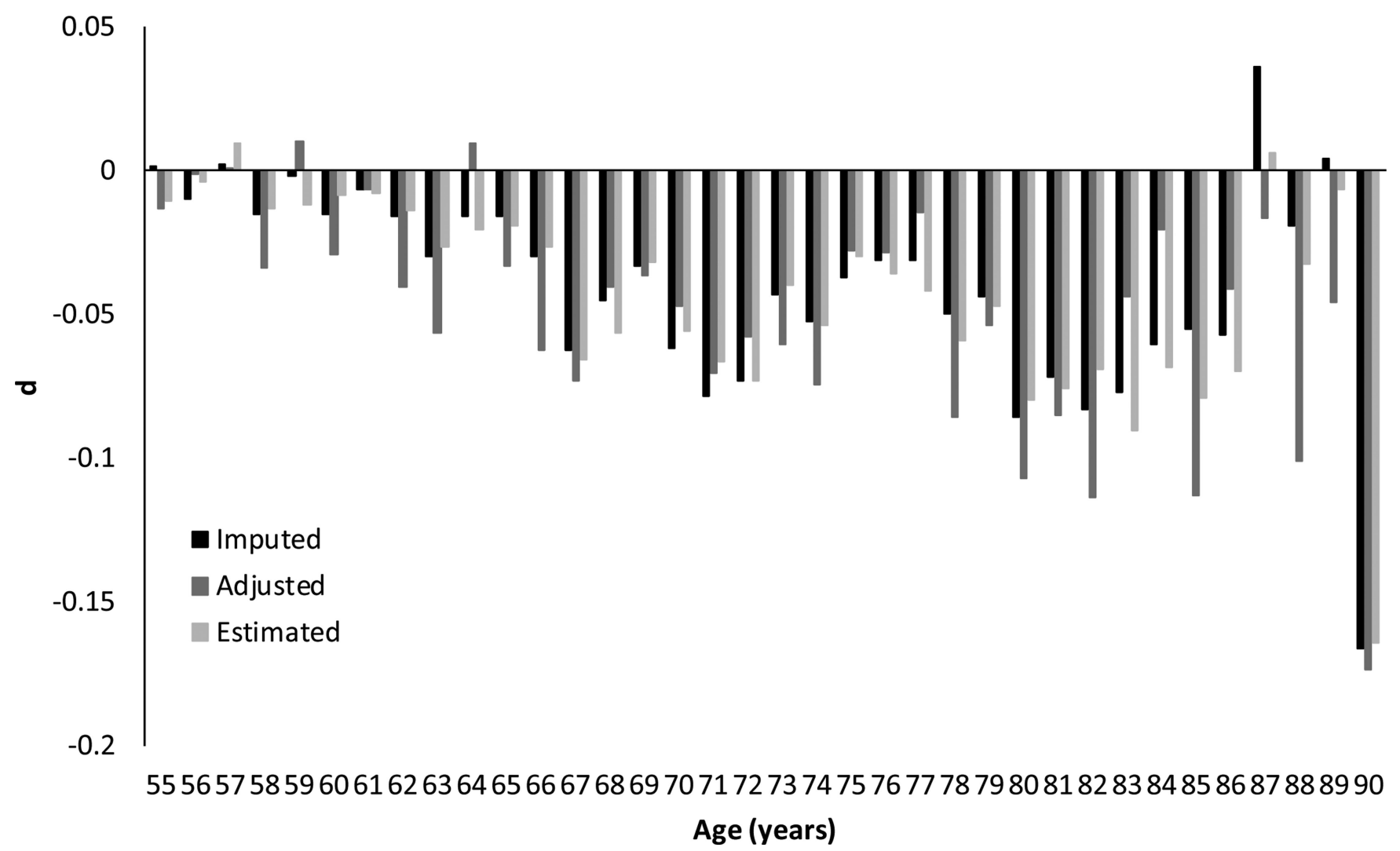

Figure 2.

Difference between observed life satisfaction and hypothetical life satisfaction when differential mortality was accounted for by imputation, estimation, or adjustment (in observed SD units $d$ ). 


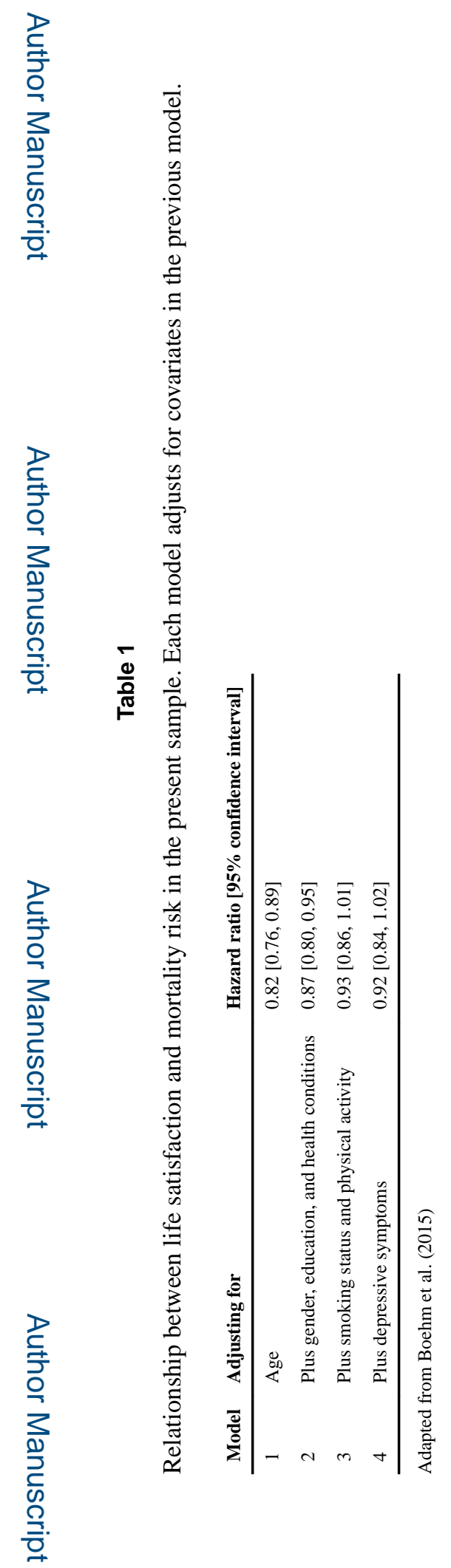

Psychol Aging. Author manuscript; available in PMC 2017 June 01. 